\title{
Comparison of Different Face Recognition Method Based On PCA
}

\section{Rajib Saha ${ }^{1}$, Debotosh Bhattacharjee ${ }^{2}$,Sayan Barman ${ }^{3}$}

${ }^{1}$ Department of Computer Science and Engineering, RCC Institute Of Information Technology, Kolkata, India

1'rajibsaha_4u@yahoo.co.in

2 Department of Computer Science and Engineering, Jadavpur University,Kolkata, India

²debotosh@indiatimes.com

${ }^{3}$ Department of Computer Science and Engineering, RCC Institute Of Information Technology, Kolkata, India

${ }^{3}$ sayanbarman84@gmail.com

\section{ABSTRACT}

In this paper, an efficient method for face recognition using principal component analysis (PCA) is presented. Nowadays PCA has been widely active for face recognition algorithms. It is one of the most popular representation methods for a face image. Face recognition involves matching a given image with the database of images and identifying the image that it resembles the most. The aim is to successfully demonstrate the human face recognition using Principal component analysis \& comparison of Manhattan distance, Euclidean distance \& Chebychev distance for face matching.

\section{Keywords}

Eigenfaces, Eigenvector, Eigenvalue, PCA, Multiview, Euclidean distance, Chebychev distance, Manhattan distance.

\section{Council for Innovative Research}

Peer Review Research Publishing System

Journal: International Journal Of Management \& Information Technology

Vol. 10, No 4

editorsijmit@gmail.com

www.ijmit.com 


\section{INTRODUCTION}

Computer model of face recognition is interesting because they can contribute not only to theoretical insight but also to practical applications. Computers that recognize faces could be applied to a wide variety of problems, including criminal identification, security systems, image processing and human-computer interaction. For example the ability to model a particular face and distinguish it from a large number of stored face models would make it possible to vastly improve criminal identification. Human face plays an important role in conveying individual's identity. According to M. A. Turk and A. P. Pentland ${ }^{[1]}$, despite large variations in the viewing conditions such as lighting, expressions, differences in hair style and so on, the human ability to recognize faces is remarkable.

Since face has complex features, developing a face recognition computer system is a very difficult task. Automated system has to address all the difficult steps involved in face recognition and detection.

Linear Discriminate Analysis algorithm is also used in face recognition. Originally developed in 1936 by R.A. Fisher, Discriminant Analysis is a classic method of classification that has stood the test of time ${ }^{[3]}$. Discriminant analysis often produces models whose accuracy approaches (and occasionally exceeds) more complex modern methods ${ }^{[3]}$. LDA is similar to Principal Component Analysis (PCA). In both PCA and

LDA there is linear combinations of the variables which explain the given data well. LDA explicitly attempts to model the difference between the classes of data ${ }^{[4]}$. PCA on the other hand does not take into account any difference in class but builds the feature combinations based on differences rather than similarities.

According to Jolliffe ${ }^{[2]}$, this difficulty is due to the fact that the faces must be represented in a way that best utilizes the available face information to distinguish a particular face from all other face. Several algorithms are used for face recognition. Some of the popular methods are discussed here. Face recognition by feature matching is one such method carried out by Manjunath ${ }^{[4]}$ et.al. In this we have to locate points in the face image with high information content. We don't have to consider the face contour or the hair. We have to concentrate on the center of the face area, as most stable and informative features are found there. The high informative points in the face are considered around eyes, nose and mouth. To enforce this we apply Gaussian weighting to the center of the face.

To identify faces in different illuminance conditions is a challenging problem for face recognition. The same person, with the same facial expression, and seen from the same viewpoint, can appear dramatically different as lighting condition changes. In recent years, two approaches, the fisherface space approach ${ }^{[6]}$ and the illumination subspace approach ${ }^{[7]}$, have been proposed to handle different lighting conditions.

The proposed scheme is based on an theory approach that decomposes face images into a small set of characteristic feature images called eigenfaces, which is a thought of as the principal components of the initial training set of face images. Recognition is performed by projecting a new image into the subspace spanned by the eigenfaces and then classifying the face by comparing its position in face space with the positions of known individuals.

In a computer based face recognition system, each face is represented by a large number of pixel values. Linear discriminant analysis is primarily used here to reduce the number of features to a more manageable number before classification. Each of the new dimensions is a linear combination of pixel values, which form a template. The linear combinations obtained using Fisher's linear discriminant are called Fisher Faces ${ }^{[3]}$.

Principal Component Analysis with Eigen faces is one of the popular algorithms for face recognition. Principal component analysis is a mathematical procedure that uses orthogonal co-ordinates to convert a set of observations of possibly correlated variables into a set of values of uncorrelated variables called principal components ${ }^{[2]}$. PCA is then applied on the Eigen faces.

Traditional 2D methods give satisfying result on frontal view or any single view. In order to extend the capability of 2D method to identifying faces in multiple views, multiview face databases are needed to train face recognition algorithm to identifying faces in different view. FRAV face database has been used in this paper for multiview faces of same person.

\section{CALCULATING EIGENFACES}

Let a face image $\Gamma(\mathrm{x}, \mathrm{y})$ be a two-dimensional $N$ by $N$ array of intensity values. An image may also be considered as a vector of dimension $N^{2}$, so that a typical image of size 256 by 256 becomes a vector of dimension 65,536 , or equivalently, a point in 65,536-dimensional space. An ensemble of images, then, maps to a collection of points in this huge space.

Images of faces, being similar in overall configuration, will not be randomly distributed in this huge image space and thus can be described by a relatively low dimensional subspace. The main idea of the principal component analysis is to find the vector that best account for the distribution of face images within the entire image space. These vectors define the subspace of face images, which we call "face space". Each vector is of length $N^{2}$, describes an $N$ by $N$ image, and is a linear combination of the original face images. Because these vectors are the eigenvectors of the covariance matrix corresponding to the original face images, and because they are face-like in appearance, they are referred to as eigenfaces $^{[5]}$. 


\section{PROPOSED METHOD}

Let the training set of face images be $\Gamma_{1}, \Gamma_{2}, \Gamma_{3}, \ldots, \Gamma_{M}$. The average face of the set if defined by $\Psi=\frac{1}{M} \sum_{n=1}^{M} \Gamma_{n}$.

Each face differs from the average by the vector $\Phi_{n}=\Gamma_{n}-\Psi$. This set of very large vectors is then subject to principal component analysis, which seeks a set of $M$ orthonormal vectors, $\mu_{n}$, which best describes the distribution of the data. The $k$ th vector, $\mu_{k}$ is chosen such that

$\lambda_{k}=\frac{1}{M} \sum_{n=1}^{M}\left(\mu_{k}^{T} \Phi_{n}\right)^{2}$

is a maximum, subject to

$\mu_{l}^{T} \mu_{k}=\left\{\begin{array}{l}1, l=k \\ 0, \text { otherwise }\end{array}\right.$

The vectors $\mu_{k}$ and scalars $\lambda_{k}$ are the eigenvectors and eigenvalues, respectively, of the covariance matrix

$C=\frac{1}{M} \sum_{n=1}^{M} \Phi_{n} \Phi_{n}^{T}=A A^{T}$

where the matrix $A=\left[\Phi_{1} \Phi_{2} \ldots \Phi_{M}\right]$. The matrix $C$, however, is $N^{2}$ by $N^{2}$, and determining the $N^{2}$ eigenvectors and eigenvalues is an intractable task for typical image sizes. A computationally feasible method is needed to find these eigenvectors.

If the number of data points in the image space is less than the dimension of the space $\left(M<N^{2}\right.$ ), there will be only $M-1$, rather than $N^{2}$, meaningful eigenvectors (the remaining eigenvectors will have associated eigenvalues of zero).

Consider the eigenvectors $v_{n}$ of $A^{T} A$ such that

$A^{T} A v_{n}=\lambda_{n} v_{n}$

Premultiplying both sides by $A$, we have

$A A^{T} A v_{n}=\lambda_{n} A v_{n}$

from which we see that $A v_{n}$ are the eigenvectors of $C=A A^{T}$.

Following this analysis, we construct the $M$ by $M$ matrix $L=A^{T} A$, where $L_{m n}=\Phi_{m}^{T} \Phi_{n}$, and find the $M$ eigenvectors $v_{n}$ of $L$. These vectors determine linear combinations of the $M$ training set face images to form the eigenfaces $\mu_{n}$ :

$\mu_{n}=\sum_{k=1}^{M} v_{n k} \Phi_{k}=A v_{n}, n=1, \ldots \ldots, M$

With this analysis the calculations are greatly reduced, from the order of the number of pixels in the images $\left(N^{2}\right)$ to the order of the number of images in the training set $(M)$. In practice, the training set of face images will be relatively small $\left(M<N^{2}\right)$, and the calculations become quite manageable. The associated eigenvalues allow us to rank the eigenvectors according to their usefulness in characterizing the variation among the images.

\section{USING EIGENFACES TO CLASSIFY A FACE IMAGE}

The eigenface images calculated from the eigenvectors of $L$ span a basis set with which to describe face images. As mentioned before, the usefulness of eigenvectors varies according their associated eigenvalues. This suggests we pick up only the most meaningful eigenvectors and ignore the rest, in other words, the number of basis functions is further reduced from $M$ to $M^{\prime}\left(M^{\prime}<M\right)$ and the computation is reduced as a consequence. 
In practice, a smaller $M^{\prime}$ is sufficient for identification, since accurate reconstruction of the image is not a requirement. In this framework, identification becomes a pattern recognition task. The eigenfaces span an $M^{\prime}$ dimensional subspace of the original $N^{2}$ image space. The $M^{\prime}$ most significant eigenvectors of the $L$ matrix are chosen as those with the largest associated eigenvalues.

A new face image $\Gamma$ is transformed into its eigenface components (projected onto "face space") by a simple operation

$\omega_{n}=\mu_{n}(\Gamma-\Psi)$

for $n=1, \ldots \ldots, M^{\prime}$. This describes a set of point-by-point image multiplications and summations.

The weights form a vector $\Omega^{T}=\left[\omega_{1}, \omega_{2}, \ldots, \omega_{M^{\prime}}\right]$ that describes the contribution of each eigenface in representing the input face image, treating the eigenfaces as a basis set for face images. The vector may then be used in a standard pattern recognition algorithm to find which of a number of predefined face classes, if any, best describes the face. The simplest method for determining which face class provides the best description of an input face image is to find the face class $k$ that minimizes the Euclidian distance

$\varepsilon_{k}^{2}=\left\|\left(\Omega-\Omega_{k}\right)^{2}\right\|$

where $\Omega_{k}$ is a vector describing the $k$ th face class. The face classes $\Omega_{k}$ are calculated by averaging the results of the eigenface representation over a small number of face images (as few as one) of each individual. A face is classified as "unknown", and optionally used to created a new face class.

\section{Design/Algorithm :}

Face recognition using Eigen faces approach was initially developed by Sirovich and Kirby and later used by Matthew Turk and Alex Pentland ${ }^{[1]}$. They showed that a collection of face images can be approximately reconstructed by storing a small collection of weights for each face and a small set of standard pictures ${ }^{[1]}$.

Using Principal Component Analysis on a set of human face images, a set of Eigen faces can be generated. Eigen faces are a set of eigenvectors used mostly in human face recognition. Eigenvectors are a set of features that characterize the variation between face images. These eigenvectors are derived from the covariance matrix of the probability distribution of the high-dimensional vector space of faces of human beings. The main idea here is to use only the best Eigen faces that account for the major variance within the set of face images. By using lesser Eigen faces, computational efficiency and speed is achieved. The Eigen faces are the basis vectors of the Eigen face decomposition.

Below are the steps of face recognition process:

- A training set of same resolution digital images is initially prepared.

- The images are stored as a matrix with each row corresponding to an image.

- Each image is represented as a vector with ( $\mathrm{X} \times \mathrm{c}$ ) elements where " $r$ " and "c" are the number of rows and the number of columns respectively.

- $\quad$ An average image is calculated from the individual training set images.

- $\quad$ For each image, the deviation from the average image is then calculated and stored.

- The Eigen vectors and Eigen values are then calculated. These represent the directions in which the training set images differ from the average image.

- A new image is then subtracted from the average image and projected into Eigen face space.

- This is compared with the projection vectors of training faces and the matching image is determined.

\section{EXPERIMENTAL RESULTS}

Here all the 2D frontal faces from FRAV3D image database are taken. As this algorithm may be applied for 3D faces in future, so 3D database is taken. The face recognition system was tested using a set of face images of 232 training images and 16 test images in total of 248 images. All the training and testing images are colour bitmap images of size $400 \times 400$. Except my own images which are of different sizes of bitmap images. For that purpose as a part of image preprocessing all the images are first converted to size of $400 \times 400$.

\section{Detailed Results :}

If the system correctly relates the test image with its correspondence in the training set, we say it conducts a true-positive identification ;

If the test image is from an unknown individual \& the system recognizes it as unknown individual, the system conducts a true -negative identification.

If the system relates the test image with a wrong person, or if the test image is from an unknown individual while the system recognizes it as one of the persons in the database, a false-positive identification is performed;

If the system identifies the test image as unknown while there does exist a correspondence between the test image and one of the training images, the system conducts a false-negative identification. 


\section{ISSN 2278-5612}

The experiment results are illustrated in the Table below:

Table 1: Recognition using Eucleadian distance

\begin{tabular}{|l|l|}
\hline Number of test images & 160 \\
\hline Number of true-positive identifications & 130 \\
\hline Number of true-negative identifications & 10 \\
\hline Number of false-positive identifications & 20 \\
\hline Number of false-negative identifications & 0 \\
\hline Percentage of images successfully matched & $87.50 \%$ \\
\hline Acceptance rate & $140 / 160$ \\
\hline Accuracy Percentage & $93.75 \%$ \\
\hline
\end{tabular}

Table 2: Recognition using Chebychev distance

\begin{tabular}{|l|l|}
\hline Number of test images & 160 \\
\hline Number of true-positive identifications & 140 \\
\hline Number of true-negative identifications & 10 \\
\hline Number of false-positive identifications & 10 \\
\hline Number of false-negative identifications & 0 \\
\hline Percentage of images successfully matched & $93.75 \%$ \\
\hline Acceptance rate & $150 / 160$ \\
\hline Accuracy Percentage & $96.87 \%$ \\
\hline
\end{tabular}

Table 3: Recognition using Manhattan distance ${ }^{[8]}$

\begin{tabular}{|l|l|}
\hline Number of test images & 160 \\
\hline Number of true-positive identifications & 140 \\
\hline Number of true-negative identifications & 10 \\
\hline Number of false-positive identifications & 10 \\
\hline Number of false-negative identifications & 0 \\
\hline Percentage of images successfully matched & $93.75 \%$ \\
\hline Acceptance rate & $15 / 16$ \\
\hline Accuracy Percentage & $96.87 \%$ \\
\hline
\end{tabular}

However, it is a long way to go before confidently drawing a conclusion on the roughness/sensitivity of the eigenfaces recognition approach. Large case study needs carrying out in the sense that:

(1) numerous tests are necessary, with face images of people who are or aren't in the database;

(2)Thresholding issue affects the performance of the algorithm. Larger threshold value leads to lower false-negative rate, but higher false-positive rate; and vice versa. In other words, a good choice of threshold value could well balance falsenegative and false-positive rates, thus maximize good recognition rate. So, it can be chosen in some proper way. 


\section{ISSN 2278-5612}

\section{Output:}

True positive recognitions:
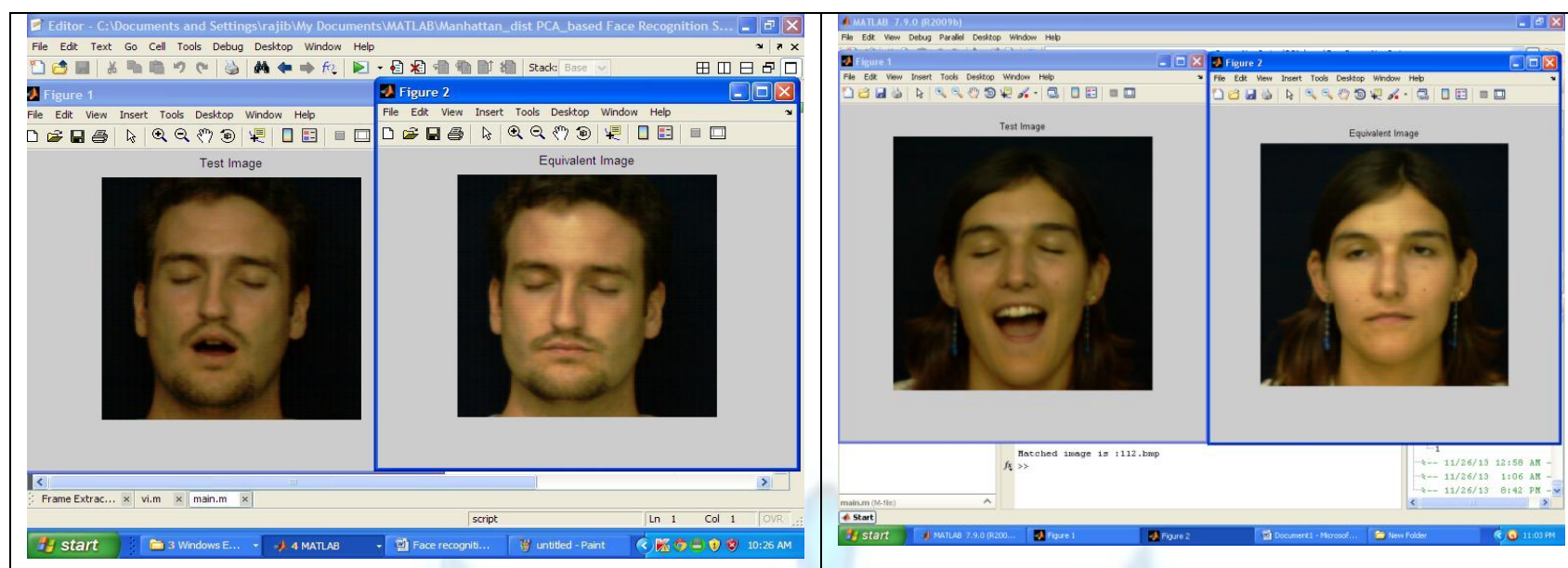

Figure : Test Result1

Figure : Test Result2
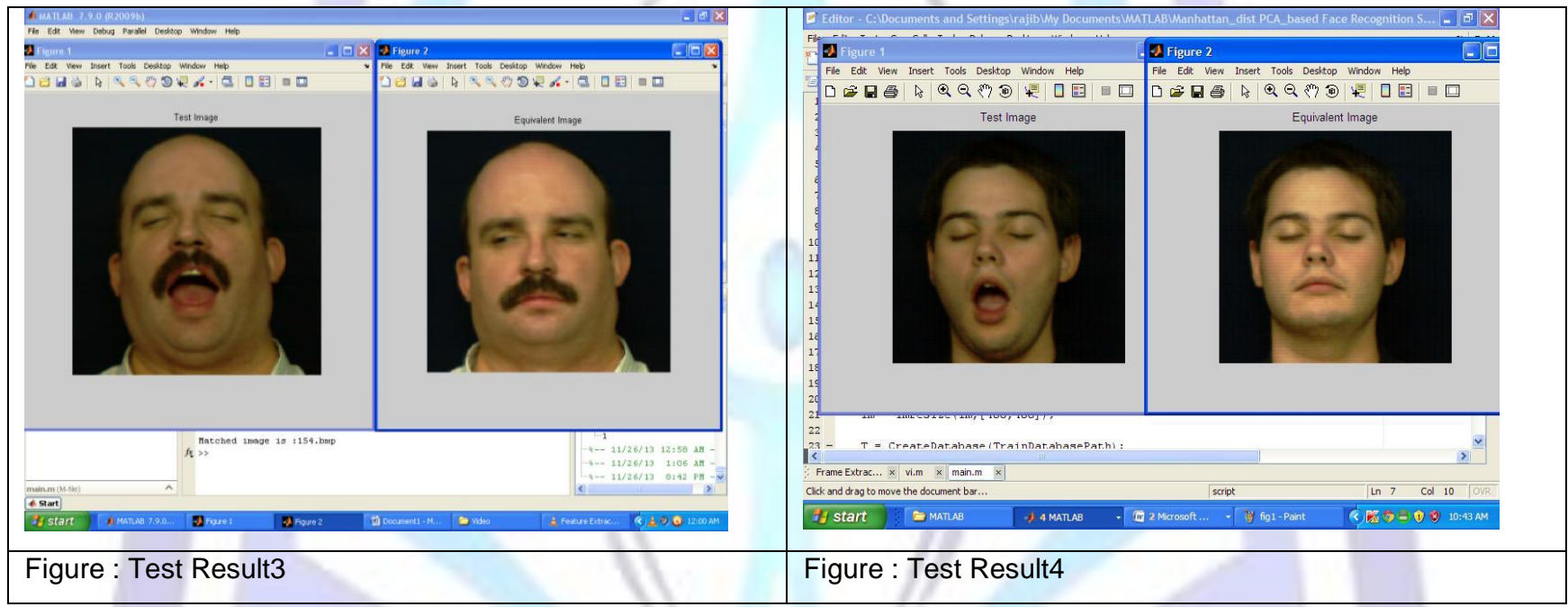

True negative recognitions:

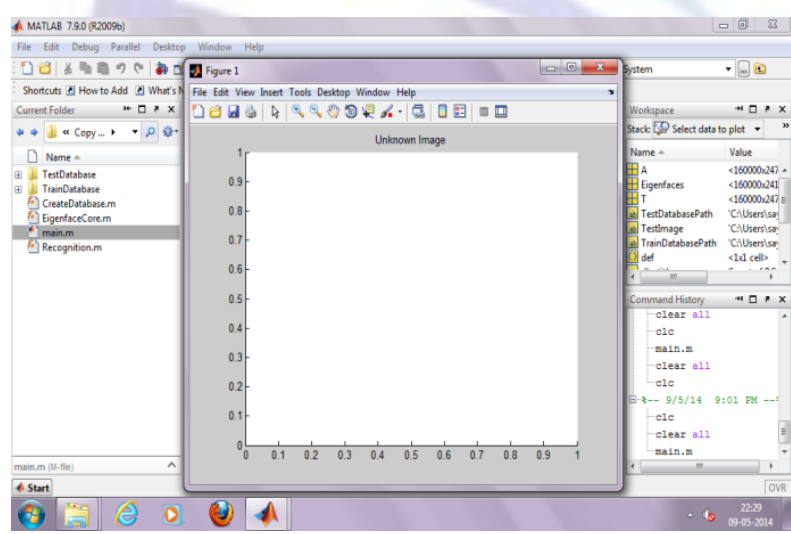

Figure : Test Result5 


\section{ISSN 2278-5612}

\section{REFERENCES}

[1] M. A. Turk and A. P. Pentland, "Face Recognition Using Eigen faces", In Proc. of Computer Vision and Pattern Recognition, pages 586-591. IEEE, June 1991.

[2] Jolliffe, I.T., "Principal Component Analysis Springer Series in Statistics", 1-9, DOI: 10.1007/0-387-22440-8_1, 2002.

[3] Peter N. Belhumeur, Joa P. Hespanha, and David J. Kriegman, "Eigen faces vs. Fisher faces: Recognition Using Class Specific Linear Projection", IEEE TRANSACTIONS ON PATTERN ANALYSIS AND MACHINE INTELLIGENCE, VOL. 19, NO. 7, JULY 1997.

[4] B.S. Manjunath, R. Chellappa, C. von der Malsburg, "A Feature Based Approach to Face Recognition”, In Proceedings, IEEE Conference on Computer Vision and Pattern Recognition, 1992.

[5] "Eigenfaces for recognition", M. Turk and A. Pentland, Journal of Cognitive Neuroscience, vol.3, No.1, 1991

[6] "Illumination cones for recognition under variable lighting: Faces", A. S. Georghiades, D. J. Kriegman, and P. N. Belhumeur, Proc. IEEE Conf. on Computer Vision and Pattern Recognition, pages 52-59, 1998.

[7] "Human face segmentation and identification", S. A. Sirohey, Technical Report CAR-TR-695, Center for Automation Research, University of Maryland, College Park, MD, 1993

[8] "Comparative study of statistical models and classifiers in face recognition"Ragul, G. ; MageshKumar, C. ; Thiyagarajan, R. ; Mohan, R. , Page(s): 623- 628, IEEE 2013.
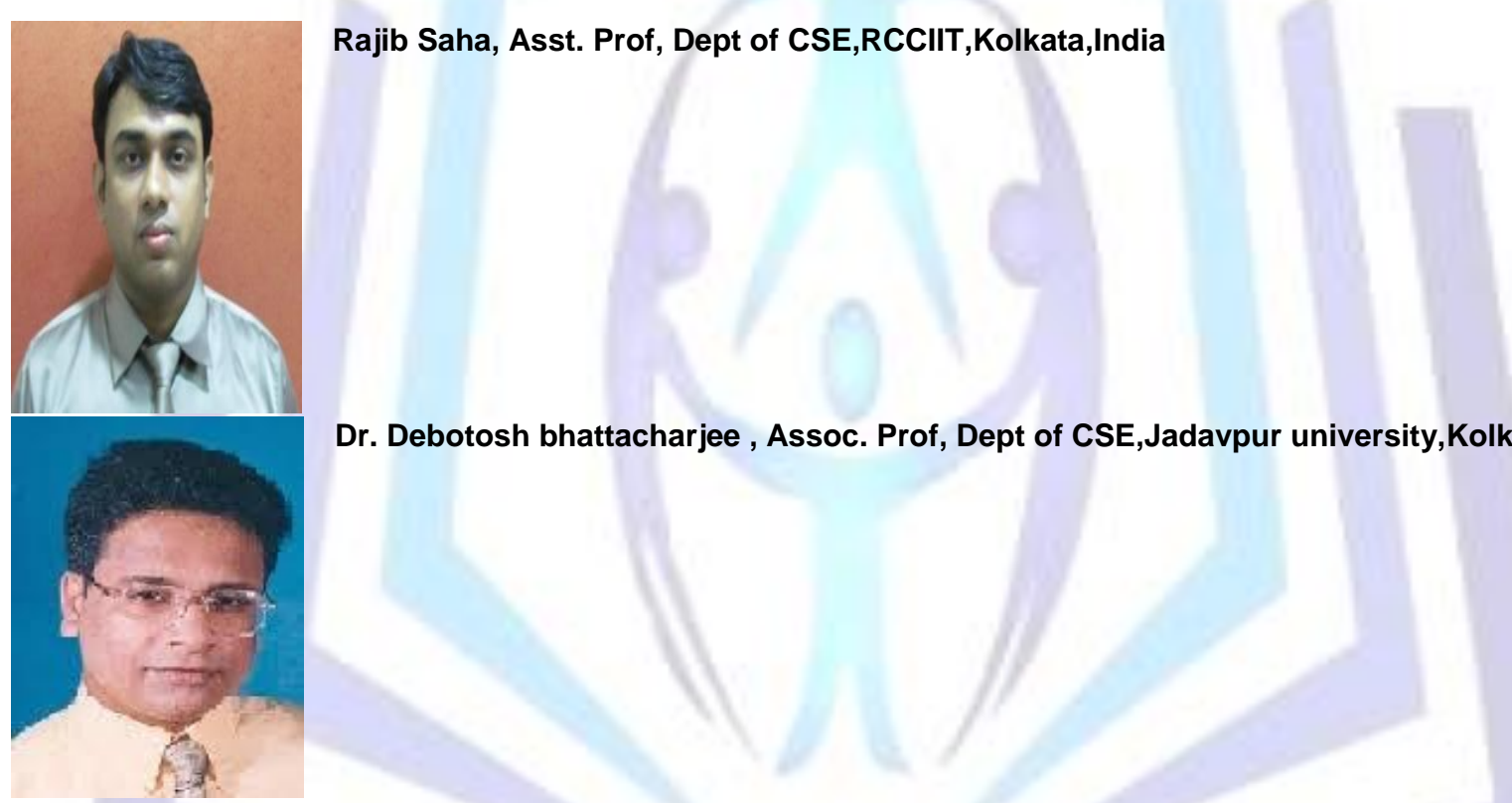

Dr. Debotosh bhattacharjee , Assoc. Prof, Dept of CSE,Jadavpur university,Kolkata,India

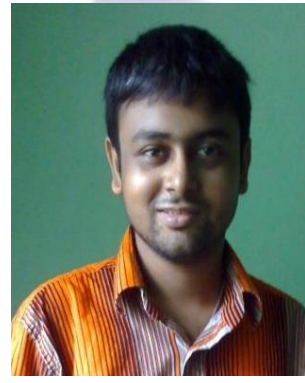

\section{Sayan Barman, Student,Dept of CSE, RCCIIT, Kolkata,India}

